\title{
Abundant molecular gas and inefficient SF in intra-cluster regions of a ram pressure stripped tail
}

\author{
P. Jáchym ${ }^{1}$, M. Sun ${ }^{2}$, F. Combes ${ }^{3}$, L. Cortese ${ }^{4}$ and J. D. P. Kenney ${ }^{5}$ \\ ${ }^{1}$ Astronomical Institute, Academy of Sciences of the Czech Republic, Boční II 1401, 14100, \\ Prague, Czech Republic, email: jachym@ig.cas.cz \\ ${ }^{2}$ Department of Physics, University of Alabama in Huntsville, 301 Sparkman Drive, Huntsville, \\ AL 35899, USA \\ ${ }^{3}$ Observatoire de Paris, LERMA, 61 Av. de l'Observatoire, 75014 Paris, France \\ ${ }^{4}$ Centre for Astrophysics \& Supercomputing, Swinburne University of Technology, Mail H30, \\ P. O. Box 218, Hawthorn, VIC 3122, Australia \\ ${ }^{5}$ Department of Astronomy, Yale University, 260 Whitney Ave., New Haven, CT 06511, USA
}

\begin{abstract}
For the first time in any ram pressure stripped galaxy, we detect large amounts of cold molecular gas in the X-ray bright, and star forming tail of ESO 137-001 in the Norma cluster. We find very low star formation efficiency in the stripped gas, similar to values found in the outer spiral disks where however molecular gas is mostly undetected. The results were recently published in Jáchym et al. (2014).
\end{abstract}

\section{Introduction}

Ram pressure of the intra-cluster medium (ICM) can strip cold, star-forming interstellar matter (ISM) reservoir from late-type galaxies in clusters (Gunn \& Gott 1972), and thus quench their star formation (SF). The stripped ISM forms one-sided 'cometary' tails that have been revealed in many galaxies in $\mathrm{HI}$, diffuse $\mathrm{H} \alpha$, young stars (seen either in $\mathrm{H} \alpha$ or UV), and X-rays (see Jáchym et al. 2014, for references). It is now understood that gas-stripped tails observed in different wavelengths are manifestations of the stripped ISM that mixed with the surrounding ICM. Part of the stripped gas can get heated to temperatures between that of HI and the ICM, while other part of the (denser) gas can efficiently cool and form cold, molecular clouds, and thus stars.

\section{Results}

With the APEX telescope, we searched for cold molecular gas, as traced by $\mathrm{CO}(2-1)$ emission, in the iconic ram pressure stripped galaxy ESO 137-001 located in the central part of the Norma cluster (A3627). This galaxy has an $80 \mathrm{kpc}$ long, bright, double structure X-ray tail, and a $40 \mathrm{kpc} \mathrm{H} \alpha$ tail with over $30 \mathrm{HII}$ regions (Sun et al. 2006, 2007, 2010). Fig. 1 shows the galaxy, together with the $\operatorname{APEX~} \operatorname{CO}(2-1)$ spectra obtained in four pointings (marked with circles). Clearly, CO emission is detected in all positions, including the most distant one at $\sim 40 \mathrm{kpc}$ downstream in the tail, where also $\mathrm{H} \alpha$ and $\mathrm{X}$-ray emission have a local peak. It is the first time that cold molecular gas is found at large distances downstream in a ram pressure stripped tail. This observation thus brings a new insight into the fate of the stripped ISM and its evolution in the intracluster space.

The corresponding total $\mathrm{H}_{2}$ mass in the tail is $\gtrsim 10^{9} \mathrm{M}_{\odot}$, assuming a Galactic value of the CO-to- $\mathrm{H}_{2}$ conversion factor. This is an amount similar to the total mass of the X-ray emitting gas. Together, they nearly account for the gas mass missing from the galaxy. The 

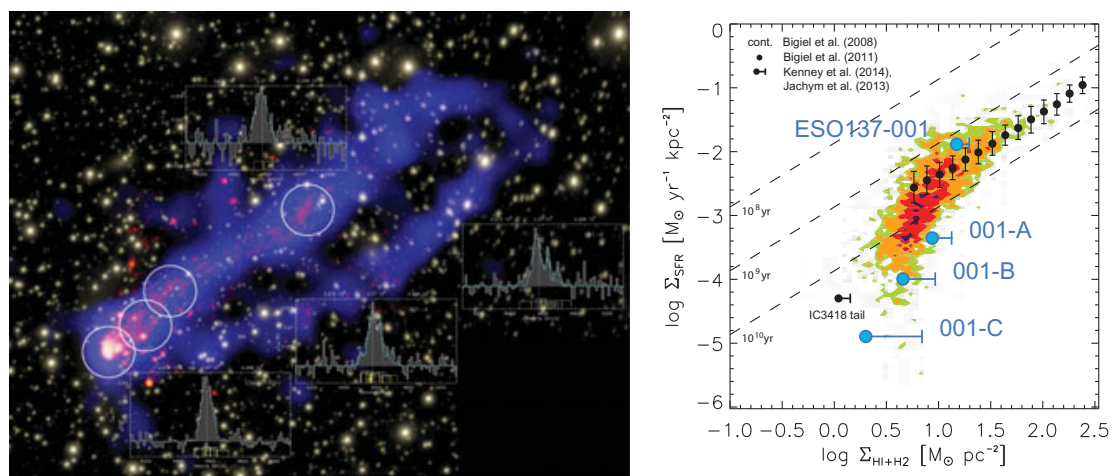

Figure 1. Left: Observed APEX positions on a composite X-ray Chandra (blue) / $\mathrm{H} \alpha$ SOAR (red) image of ESO 137-001. The FWHM of the $\mathrm{CO}(2-1)$ beam is $25 " \approx 8.4$ kpc. Right: SFR surface density as a function of total gas surface density in ESO 137-001 and the three observed tail regions 001-A, 001-B, and 001-C. The filled circles correspond to $\mathrm{H}_{2}$ column density, while the 'error bars' show ATCA HI upper limits. Figure adapted from Bigiel et al. (2008). From Jáchym et al. (2014).

$\mathrm{H} \alpha$-based star formation rates (SFRs) averaged over the APEX beams (following Kennicutt \& Evans 2012) are for the observed pointings placed into the Schmidt-Kennicutt-type plot in Fig. 1. While in the main body of the galaxy, the measured molecular gas depletion time is well consistent with average values found in nearby galaxies, clearly, the location of the tail points indicates much lower efficiency of star formation (SFE) in the stripped gas than is typical in star-forming ISM in the inner parts of spiral disks.

\section{Conclusions}

The measured low SFE $\left(\tau_{\mathrm{dep}, \mathrm{H}_{2}}>10^{10} \mathrm{yr}\right)$ in the tail of ESO 137-001 suggests that most of the stripped gas does not form stars but ultimately joins the ICM. Similarly low SFEs are found in the outer disks of spiral galaxies, where however, HI is likely the dominant component of the ISM and CO is mostly undetected (e.g., Bigiel et al. 2010). This might be due to low volume density of the stripped gas, and/or ram pressure induced shocks that may increase thermal and turbulent pressure of the stripped ISM. The CObright tail of ESO 137-001 thus represents a special environment of star formation.

The detection of abundant molecular gas in the X-ray bright tail of ESO 137-001 suggests that in massive, high-ICM-pressure clusters (such as Norma), the conditions are right for the mixing of the stripped ISM with the surrounding ICM to produce observable levels of individual gas components. This motivates further multi-wavelength studies of ram pressure stripped galaxies in rich clusters that would allow us to reveal more of the stripped ISM and thus to learn about its fate and evolution in the intra-cluster space.

\section{Acknowledgements}

We acknowledge support from the project M100031203 of the Academy of Sciences of the Czech Republic.

\section{References}

Bigiel, F., Leroy, A., Walter, F., et al. 2010, AJ, 140, 1194

Bigiel, F., Leroy, A., Walter, F., et al. 2008, AJ, 136, 2846 
Gunn, J. E. \& Gott, III, J. R. 1972, ApJ, 176, 1

Jáchym, P., Combes, F., Cortese, L., et al. 2014, ApJ, 792, 11

Kennicutt, R. C. \& Evans, N. J. 2012, ARA\&A, 50, 531

Sun, M., Donahue, M., Roediger, E., et al. 2010, ApJ, 708, 946

Sun, M., Donahue, M., \& Voit, G. M. 2007, ApJ, 671, 190

Sun, M., Jones, C., Forman, W., et al. 2006, ApJ, 637, L81 